DPNU-03-13

hep-th/0305130

\title{
On the solutions to accelerating cosmologies
}

\author{
Masato Ito * \\ Department of Physics, Nagoya University, Nagoya, JAPAN 464-8602
}

\begin{abstract}
Motivated by recent accelerating cosmological model, we derive the solutions to vacuum Einstein equation in $(d+1)$-dimensional Minkowski space with $n$ dimensional hyperbolic manifold. The conditions of accelerating expansion are given in such a set up.
\end{abstract}

\footnotetext{
*E-mail address: mito@eken.phys.nagoya-u.ac.jp
} 
Recently, Townsend and Wohlharth discovered a solution of the four-dimensional accelerating cosmologies with compact hyperbolic manifold [1]. By avoiding the assumptions of a no-go theorem [2], it was pointed out that the accelerating phase arises in a interval. The features of the model are that the four-dimensional metric is Einstein frame and the compact hyperbolic manifold is time-dependent. Before [1], the investigations of FRW universes with closed hyperbolic internal space have been widely performed $[3,4,5,6,7]$. Furthermore it has been shown that the model is closely connection with the S-branes which correspond to time-dependent solutions of supergravity with $p$-form $[8,9,10,11,12,13,14]$. Thus it is expected that the accelerating universe supported by astronomical observations may be explained by string/M-theory.

From the theoretical point of view, we are interested in the higher dimensional cosmological model motivated by the model of Ref.[1]. In this letter, we derive the solutions to the vacuum Einstein equation in time-dependent $\mathcal{M}_{d+1} \times H_{n}$, where $\mathcal{M}_{d+1}$ and $H_{n}$ are $(d+1)$-dimensional Minkowski and $n$-dimensional hyperbolic manifold, respectively. Furthermore we investigate the conditions of accelerated expansion of the present model.

Adopting the Einstein frame metric for $d \geq 3$, we can take ansatz for timedependent metric

$$
d s^{2}=b^{\frac{4}{d-1}}(t) d s_{d+1}^{2}+b^{-\frac{4}{n}}(t) d \hat{s}_{n}^{2}
$$

where

$$
d s_{d+1}^{2}=-a^{2 d}(t) d t^{2}+a^{2}(t) d x_{d}^{2}
$$

where $a(t)$ corresponds to the scale factor of $d$-dimensions. For the $n$-dimensional hyperbolic manifold, the Ricci tensor is given by

$$
\hat{\mathcal{R}}_{a b}=-(n-1) g_{a b} .
$$

The Ricci tensors for metric (1) are evaluated as follows,

$$
\begin{aligned}
\mathcal{R}_{t t} & =-\frac{2}{d-1} \frac{\ddot{b}}{b}-2\left(\frac{1}{d-1}+\frac{2}{n}\right) \frac{\dot{b}^{2}}{b^{2}}-d \frac{\ddot{a}}{a}+d^{2} \frac{\dot{a}^{2}}{a^{2}} \\
\mathcal{R}_{\mu \nu} & =a^{2(1-d)}\left\{\frac{2}{d-1}\left(\frac{\dot{b}}{b}\right)+\left(\frac{\dot{a}}{a}\right)\right\} g_{\mu \nu}
\end{aligned}
$$




$$
\mathcal{R}_{a b}=\left[-\frac{2}{n} b^{-4\left(\frac{1}{n}+\frac{1}{d-1}\right)} a^{-2 d}\left(\frac{\dot{b}}{b}\right)-(n-1)\right] g_{a b}
$$

where $\mu, \nu$ and $a, b$ represent $\mathcal{M}_{d+1}$ and $H_{n}$, respectively. The dot denotes the derivative with respect to the time $t$. In order to simplify the vacuum Einstein equation for metric ansatz, we define variables $X$ and $Y$ by

$$
\begin{aligned}
& b=e^{X} \\
& a=e^{Y} .
\end{aligned}
$$

Using the new variables, the equations of motion can be rewritten as

$$
\begin{aligned}
-\frac{2}{d-1} \ddot{X}-4\left(\frac{1}{d-1}+\frac{2}{n}\right) \dot{X}^{2}-d \ddot{Y}+d(d-1) \dot{Y}^{2} & =0, \\
\frac{2}{d-1} \ddot{X}+\ddot{Y} & =0, \\
\ddot{X}+\frac{1}{2} n(n-1) e^{4\left(\frac{1}{d-1}+\frac{1}{n}\right) X+2 d Y} & =0 .
\end{aligned}
$$

Performing the integral of (7), and we have

$$
\frac{2}{d-1} X+Y=f t+g
$$

where $f$ and $g$ are integration constants. Substituting (9) into (6) and (8), the equations of motion can be written in terms of $X$

$$
\begin{array}{r}
\ddot{X}+2 \frac{n-1}{n} \dot{X}^{2}-2 f d \dot{X}+\frac{1}{2} d(d-1) f^{2}=0 \\
\ddot{X}=-\frac{1}{2} n(n-1) e^{2 g d} e^{-4 \frac{n-1}{n} X+2 d f t},
\end{array}
$$

In order to solve (11), we define $Z$ by

$$
Z=-4 \frac{n-1}{n} X+2 d f t
$$

Consequently, the second order differential equation can be obtained

$$
\ddot{Z}=2(n-1)^{2} e^{2 g d} e^{Z}
$$

Performing the integral of the above equation, we have

$$
\dot{Z}^{2}=4(n-1)^{2} e^{2 g d} e^{Z}+h
$$


where $h$ is positive integration constant. Therefore, the solution to the $Z$ is given by

$$
e^{Z}=\frac{h}{4(n-1)^{2}} \frac{e^{-2 g d}}{\sinh ^{2} \frac{\sqrt{h}}{2}\left(t+t_{0}\right)},
$$

where $t_{0}$ is the integration constant. Furthermore, (12) can be rewritten in terms of $Z$

$$
2 n \ddot{Z}-n \dot{Z}^{2}+4 f^{2} d(n+d-1)=0
$$

Substituting (13) and (14) into (16), the relation between the integration constants $h$ and $f$ can be expressed as

$$
h=4 \frac{d(n+d-1)}{n} f^{2} .
$$

Here we simply take $f=1, g=0, t_{0}=0$. Combining with (9), (12) and (15), and we have

$$
\begin{aligned}
& b=e^{X}=e^{\frac{n d}{2(n-1)} t}\left(\frac{\gamma}{(n-1) \sinh \gamma|t|}\right)^{-\frac{n}{2(n-1)}} \\
& a=e^{Y}=e^{-\frac{n+d-1}{(n-1)(d-1)} t}\left(\frac{\gamma}{(n-1) \sinh \gamma|t|}\right)^{\frac{n}{(d-1)(n-1)}}
\end{aligned}
$$

where

$$
\gamma=\sqrt{\frac{d(n+d-1)}{n}}
$$

Note that the argument of the hyperbolic sine can be taken to be positive in order that the solutions are real.

For $d=3$, obviously, (18) and (19) are completely consistent with the metric in Ref.[1]. Furthermore it is considered that the results obtained here are included in S-brane solutions in arbitrary dimensions derived by N. Ohta [10].

Below we shall investigate expansion and acceleration in the case of $d \geq 3$. From the metric (2), the proper time $\eta$ for $(d+1)$-dimensional world is given by $d \eta=a^{d} d t$. The condition of expanding $(d+1)$-dimensional universe, $d a / d \eta>0$, leads to

$$
M(t)=1+\sqrt{\frac{n d}{n+d-1}} \operatorname{coth}\left(\sqrt{\frac{d(n+d-1)}{n}} t\right)<0 .
$$

The above inequality is satisfied for negative $t$ since the coefficient of hyperbolic cotangent is always larger than one. 
Moreover the condition of accelerating $(d+1)$-dimensional universe, $d^{2} a / d \eta^{2}>0$, leads to

$$
M^{2}(t)<\frac{d(n-1)}{n+d-1} \frac{1}{\sinh ^{2}\left(\sqrt{\frac{d(n+d-1)}{n}} t\right)} .
$$

The accelerating phase arises if the conditions (21) and (22) are simultaneously satisfied at negative $t$. As for the asymptotic relationship between time $t$ and proper time $\eta$, note that $t \rightarrow-\infty$ corresponds to $\eta \rightarrow+0$ while $t \rightarrow-0$ corresponds to $\tau \rightarrow \infty$. Furthermore the asymptotic behavior of scale factor for $\eta$ is expressed as

$$
a(\eta) \sim\left\{\begin{array}{ll}
\eta^{\frac{1}{d}} & \text { for } \eta \rightarrow+0(t \rightarrow-\infty) \\
\eta^{\frac{n}{n+d-1}} & \text { for } \eta \rightarrow \infty(t \rightarrow-0)
\end{array} .\right.
$$

For small $\eta$, note that scale factor is independent of $n$.

The interval of accelerating expansion is determined by $d^{2} a / d \eta^{2}=0$. Therefore we get

$$
\begin{aligned}
& t_{1}=-\sqrt{\frac{n}{d(n+d-1)}} \log \frac{\sqrt{n-1}(\sqrt{d}+1)}{\sqrt{n d}-\sqrt{n+d-1}} \\
& t_{2}=-\sqrt{\frac{n}{d(n+d-1)}} \log \frac{\sqrt{n-1}(\sqrt{d}-1)}{\sqrt{n d}-\sqrt{n+d-1}},
\end{aligned}
$$

where $t_{1}$ and $t_{2}$ are start and end of acceleration, respectively. Using the above results, we can estimate the e-foldings number in an interval of acceleration, namely, $\log \left(a\left(t_{2}\right) / a\left(t_{1}\right)\right)=\mathcal{O}(1)$. As indicated in Ref.[15], since the e-foldings number is of order one, it is questionable whether the present model is worthy of inflation model. It is expected that models or mechanisms for enlarging e-foldings number are proposed in the future.

In summary, we derived the solutions to vacuum Einstein equation in $\mathcal{M}_{d+1} \times H_{n}$ with Einstein frame metric for $d \geq 3$, triggered by recent accelerating cosmological model of Ref.[1]. We provided the conditions of accelerating expansion and the asymptotic behaviors of scale factor. Moreover, the start and end of acceleration are given and it was pointed out that the e-foldings number during the interval is approximately of order one for proper values of $n, d$. 


\section{Acknowledgements:}

We thank N. Ohta for pointing out errors in the earlier version of the paper and useful correspondence.

Note added: After we submitted this paper to the hep archives, we learned the higher dimensional accelerating cosmologies with product spaces proposed by C-M. Chen et al.[15]. It was pointed out that the model considered here is related to the S-branes by N. Ohta [10].

\section{References}

[1] P. K. Townsend and M. N. Wohlfarth, "Accelerating cosmologies from compactification," arXiv:hep-th/0303097.

[2] J. M. Maldacena and C. Nunez, "Supergravity description of field theories on curved manifolds and a no go theorem," Int. J. Mod. Phys. A 16, 822 (2001) [arXiv:hep-th/0007018].

[3] G. D. Starkman, D. Stojkovic and M. Trodden, "Large extra dimensions and cosmological problems," Phys. Rev. D 63, 103511 (2001) [arXiv:hep-th/0012226].

[4] N. Kaloper, J. March-Russell, G. D. Starkman and M. Trodden, "Compact hyperbolic extra dimensions: Branes, Kaluza-Klein modes and cosmology," Phys. Rev. Lett. 85, 928 (2000) [arXiv:hep-ph/0002001].

[5] G. D. Starkman, D. Stojkovic and M. Trodden, "Homogeneity, flatness and 'large' extra dimensions," Phys. Rev. Lett. 87, 231303 (2001) [arXiv:hep-th/0106143].

[6] P. J. Silva, "Stabilization of hyperbolic brane-world scenarios," arXiv:hepth/0202160.

[7] S. Nasri, P. J. Silva, G. D. Starkman and M. Trodden, "Radion stabilization in compact hyperbolic extra dimensions," Phys. Rev. D 66, 045029 (2002) [arXiv:hep-th/0201063].

[8] M. Gutperle and A. Strominger, "Spacelike branes," JHEP 0204, 018 (2002) [arXiv:hep-th/0202210]. 
[9] M. Kruczenski, R. C. Myers and A. W. Peet, "Supergravity S-branes," JHEP 0205, 039 (2002) [arXiv:hep-th/0204144].

[10] N. Ohta, "Intersection rules for S-branes," Phys. Lett. B 558, 213 (2003) [arXiv:hep-th/0301095].

[11] N. Ohta, "Accelerating cosmologies from S-branes," arXiv:hep-th/0303238.

[12] N. Ohta, "A study of accelerating cosmologies from superstring / M theories," arXiv:hep-th/0304172.

[13] R. Emparan and J. Garriga, "A note on accelerating cosmologies from compactifications and S-branes," arXiv:hep-th/0304124.

[14] M. N. Wohlfarth, "Accelerating cosmologies and a phase transition in M-theory," arXiv:hep-th/0304089.

[15] C. M. Chen, P. M. Ho, I. P. Neupane and J. E. Wang, "A note on acceleration from product space compactification," arXiv:hep-th/0304177. 